\title{
La Réception historique et imaginaire dans Samarcande d'Amin Maalouf
}

\author{
Dr.Kamel Mounir
}

Faculté des Lettres Menoufeya

\section{Résumé}

Cet article se propose de montrer l'univers historique et imaginaire dans Samarcande (1988) d'Amin Maalouf. Pour préciser la position de Maalouf par rapport à l'histoire de l'Orient, nous pouvons dire que lorsqu'il parle de l'histoire d'un pays, d'un homme ou d'un poète, il met en lumière son univers imaginaire. Notre article a pour objectif d'analyser la conception de la réception historique et imaginaire à travers la narration historique, ce qui nous permet de comprendre l'interférence culturelle entre l'Orient et l'Occident. Et à notre sens, quelques notions clés interviennent dans Samarcande, à savoir la tolérance, le vin, la poésie et la violence. Notre article s'inscrit dans une perspective consistant à proposer des solutions aux problématiques culturelles, idéologiques et philosophiques. Ce qui nous intéresse ici, c'est de tenter de comprendre comment Maalouf nous a offert une combinaison équilibrée entre le sens historique et le sens fictif. Nous allons Remarquer que Maalouf a réussi à construire son monde narratif, réel et fictionnel. Enfin, nous allons aborder la structure Romanesque dans Samarcande d'Amin Maalouf qui s'intéresse essentiellement aux sources historiques de l'Orient.

Mots clés: réception historique, réception imaginaire, histoire de l'Orient et de l'occident, interculturalité 


\section{Introduction}

Parmi les écrivains francophones d'origine arabe, Amine Maalouf (1949) se considère comme un médiateur entre l'Orient et l'Occident. II se trouve au carrefour des deux cultures, arabe et occidentale. II s'est consacré à la littérature après l'exil qui a amené Maalouf à entrer de plein pied dans l'univers romanesque et imaginaire. Ceci dit, il convient de remarquer que les Croisades vues par les Arabes est le premier roman écrit par Amin Maalouf. II a été publié pour la première fois en 1983

L'œuvre d'Amin Maalouf représente, en effet, la base de son univers romanesque qui s'appuie sur un imaginaire nourri de l'histoire et de la diversité culturelle (libanaise, égyptienne) et religieuse (chrétienne et musulmane). Puisque nous proposons d'étudier l'univers historique et imaginaire dans Samarcande ${ }^{1}$ (1988) d'Amin Maalouf, nous constatons que l'auteur retourne au passé pour retracer un paysage historicoimaginaire.

Samarcande n'est pas seulement un roman historique, mais c'est un roman imaginaire d'inspiration historique parce que l'histoire pour Amin Maalouf est une source d'inspiration pour penser la ville légendaire, culturelle et commerciale. C'est la cause pour laquelle Amin Maalouf a réussi à reconstruire l'identité arabe à partir de l'identité culturelle en stimulant l'imagination du lecteur. ${ }^{2}$

1 - La ville historique de Samarcande représente un Carrefour et un lieu de synthèse des cultures du monde entier. Cette ville est fondée au VIIe siècle et a connu son apogée à l'époque qui s'étend du XIVe au XVe siècle. La ville historique de Samarcande est située dans une grande oasis dans le nord-est de l'Ouzbékistan. Les arts de l'Islam ont exercé une influence notable dans cette région. La ville moderne s'est constituée autour du quartier historique.

2- «L'identité culturelle est ce par quoi se reconnaît une communauté humaine (sociale, politique, régionale, nationale, ethnique, religieuse,) en termes de valeurs, de pensées et d'engagement, de langue et de lieu de vie, de pratiques, de traditions et de croyances, de vécu en commun et de mémoire historique.)> Disponible sur https://www.grainesdepaix.org/fr/ ressources/dictionnaire/identite_culturelle. 
Dans cet article, nous suivons une démarche permettant de faire le point sur une compréhension plus précise de trois thèmes essentiels : la réception littéraire, la réception historique et la réception imaginaire. Ces thèmes nous incitent à réfléchir sur l'interaction réciproque entre l'auteur, d'un côté, et le lecteur de l'autre. Nous essaierons de dégager, à partir de l'analyse descriptive de ces deux thèmes, les caractéristiques clés de la stratégie romanesque chez Maalouf.

En fait, notre approche d'analyse descriptive permet de décrire une histoire d'une ville dans laquelle Maalouf parle de l'univers historique et imaginaire dans Samarcande. Nous nous attacherons, dans cette perspective, à étudier quelques extraits de son roman intitulé Samarcande. Cet article présente des enjeux et défis spécifiques que le chercheur rencontre lorsqu'il analyse ce texte romanesque.

\section{La réception littéraire ${ }^{3}$ conçue comme un concept théorique}

Dans un premier temps, notre propos consiste à montrer, dans les limites de cet article que la théorie de la réception littérature insiste sur le fait qu'un texte doit être lu par un lecteur. Cela veut dire que cette réception littéraire, qui représente une expérience à la fois individuelle et collective, s'intéresse à étudier le rapport entre le lecteur et l'œuvre

3- «En histoire de l'art comme en littérature, la notion de « réception » recouvre des objets et des méthodes variés, relevant de traditions intellectuelles distinctes. Le terme lui-même renvoie à l'« esthétique de la réception », développée dans le domaine de la littérature à partir des années 1960, au sein de l'école de Constance, par l'universitaire allemand Hans Robert Jauss.») Cf. RÉCEPTION, art et littérature, Disponible sur https://www.universalis.fr/encyclopedie/ réception-art-et-littérature.

" L'histoire de la réception doit donc saisir "le système de références objectivement formulable" qui entoure chaque œuvre "au moment de l'histoire où elle apparaît. Ce système est constitué par trois éléments : l'expérience préalable que le public a du genre [...], la forme et la thématique d'œuvres antérieures [...], et l'opposition entre langage poétique et langage pratique. \) Cf. Rosmarin HEIDENREICH, La problématique du lecteur et de la réception, Cahiers de recherche sociologique, no 12, printemps 1989, Disponible sur https://www.erudit.org/fr/revues/crs/1989-n12-crs1516226/1002059ar.pdf. 
littéraire. A ce propos, nous ne pouvons pas séparer le lecteur de la dimension historico-imaginaire dans le texte littéraire.

Pour sa part, Robert Jauss a remarqué que «l'esthétique de la réception n'est qu'une réflexion méthodologique partielle, susceptible d'être associée à d'autres.») (Jauss, 1978, p. 244)

Cette vision méthodologique, selon Jauss, met l'accent sur l'esthétique de la réception qui ne réside pas seulement dans sa fonction esthétique mais aussi dans l'effet qu'elle produit et qui adjoint à l'autre. Si l'écrivain est celui qui produit l'œuvre littéraire, le lecteur est un récepteur efficace qui vit dans l'attente de recevoir ce texte.

R.Jauss décrit cette attitude comme un horizon d'attente littéraire et social. II évoque également la réception passive et la réception active en citant "l'horizon ne cesse de changer, où s'opère en permanence le passage de la réception passive à la réception active, de la simple lecture à la compréhension critique, de la norme esthétique admise à son dépassement par une production nouvelle.» (Jauss, 1978, p. 45)

Cela veut dire que le texte littéraire est conditionné par une double réception passive ou active qui provient d'une lecture simple et qui contribue à une lecture compréhensible. Dans ces conditions de lecture, nous remarquons que le lecteur, qui est un récepteur fictif, a toujours soumis, à la fois, à une réalité historique et à une dimension imaginaire.

Apres avoir étudié le concept de la réception littéraire, nous constatons que le texte sert à évoquer chez le lecteur une conscience historique et imaginaire qui lui permet de découvrir le message explicite et implicite du texte littéraire. En outre, il est remarquable que l'interaction sociale désigne un échange entre le texte et la lecture active, ce qui fait de cette lecture une des phases importantes de la réception littéraire. Cette 
notion développée dans la phase de la lecture apporte un éclairage sur le rapport entre le lecteur, c'est-à-dire le récepteur actif, et l'œuvre littéraire. Ajoutons que l'engagement du lecteur dans l'acte de lecture provoque chez lui une expérience socioculturelle.

Émery-Bruneau montre que le statut de lecteur ressemble à «une personne, engagée dans une pratique de lecture littéraire, qui se construit et se transforme par son investissement sensible et réflexif dans chaque activité de lecture, et par la façon dont elle l'exprime.ı (Bruneau, 2010, p.57)

Cette citation permet de comprendre que la réception littéraire n'est pas seulement une pratique de lecture littéraire mais aussi une pratique socioculturelle. Quant à Josias Semujanga, il a souligné que «Le terme auquel est associée la théorie de la réception évoque deux phénomènes littéraires :

«la lecture et la notion de valeur des textes dits littéraires. Par ailleurs, c'est parce qu'elle privilégie le rôle du sujet lecteur dans la compréhension des œuvres, tout en insistant sur la variabilité de la notion même de valeur littéraire ou artistique, que la théorie de la réception semble concilier les deux grandes tendances opposées de la critique littéraire: la sociologie de la littérature et les méthodes structurales.») (Protée, 1999, p.4)

La lecture et la notion de textes littéraires, selon J.Samuhanga, appartiennent à deux phénomènes figuratifs de la réception littéraire. Remarquons que le sujet lecteur représente à la fois la notion de la littérature qui se considère comme un fait social et en même temps l'analyse structurale approprié à un discours qui est une pratique sociale. En plus, le mot, le texte, et l'image qui sont des produits figuratifs transmettent le sens au lecteur. 
J.Samuhanga insiste également sur la sociologie de la littérature qui permet d'orienter les lecteurs, leurs valeurs, leurs goûts et leurs attentes vers la réception des œuvres littéraires. C'est ainsi que la réception littéraire se fonde sur le système social et non pas seulement sur l'interprétation individuelle du lecteur. Comme avec les points de vue exprimés par Jauss, nous remarquons aussi qu'lser et Bakhtine sont les grands théoriciens de la réception littéraire qui exercent une influence grandissante sur la théorie littéraire.

Pour s'acquitter de sa tâche, Richard Hodgson, dans un article intitulé Mikhaïl Bakhtine et la théorie littéraire contemporaine, a constaté que " sur le plan théorique, la pensée de Bakhtine se situe au carrefour de plusieurs grands courants de la critique littéraire moderne: stylistique, sémiotique, histoire des mentalités. Comme la plupart des grands théoriciens de la littérature de ce siècle, l'auteur de: Esthétique et théorie du roman a élaboré des thèses qui relèvent directement des sciences humaines, notamment de la linguistique et de la sociologie» (Hodgson, 1995, p.49)

Nous remarquons aussi que la réception littéraire pour Mikhaïl Bakhtine est un lieu de rencontre et d'interaction entre l'auteur et le lecteur, de même que la structure de l'œuvre est un lieu de rencontre et d'interaction entre la forme et le fond du texte.

Nicolas Xanthos a mis en évidence les défis la théorie de la réception dans l'œuvre de Wolfgang Iser et Hans Robert Jauss en affirmant qu'il y a sept questions différentes englobées par la problématique de la réception :

«l'histoire littéraire en tant que "histoire propre" grâce à l'activité réceptive des lecteurs comme facteurs constitutifs du processus historique»; «la différentiation entre divers types de réception»; «la 
stratégie de réception et d'effet»; "l'appropriation active et, par conséquent, transformatrice de l'œuvre de la part du lecteur»; «l'interaction dialectique entre l'œuvre en tant que modèle ou projet de réception et le lecteur en tant que récepteurtransformateur»; "les conséquences de la réception, voire la "fonction sociale" que l'œuvre littéraire assume» (Xanthos, 1999, p.35)

Nous remarquons donc que l'activité réceptive doit pointer du doigt sur l'histoire littéraire comme facteur du processus historique, et ce pour instaurer l'interaction entre l'œuvre et le lecteur qui réside dans le cœur de la perspective historico-imaginaire.

Pour mieux comprendre cette perspective, nous allons reconnaître l'intérêt de cette approche pour adapter la réception historique et imaginaire dans Samarcande aux figures historiques. C'est la raison pour laquelle notre première réflexion, dans cet article, se porte sur le thème de la réception littéraire, ce qui nous permet d'accorder la primauté à la perception de l'auteur qui écrit le texte et à celle de lecteur qui reçoit le même texte. Quoique notre article ne retrace pas la progression des travaux ayant porté sur des théories littéraires à ce sujet, notre approche qui reflète une certaine évolution de la pensée narrative à l'égard de la réception littéraire.

A ce propos, Wolfgang a constaté que « Le texte n'existe que par l'acte de constitution d'une conscience qui la reçoit.) ${ }^{4}$ (Wolfgang, 1976 : p. 49)

4- karima Yahia Ouahmed, Etude des textes littéraires, «De ce fait, le texte n'existe que par l'acte de constitution d'une conscience qui la reçoit, et ce n'est qu'au cours de la lecture que l'œuvre acquiert son caractère particulier de processus. Désormais on ne devrait plus parler d'œuvre que lorsqu'il y a, de manière interne au texte, processus de constitution de la part du lecteur. L'œuvre est ainsi la constitution de la conscience du lecteur.>) Disponible sur 
Rappelons que le thème de la réception littéraire nous permet de dégager l'image que le lecteur peut avoir de la création de notre romancier, et ce dans le but d'aborder les principes régissant l'acte de lecture selon l'approche choisie. Nous constatons alors que le romancier qui écrit le texte littéraire et le lecteur qui réagit en lisant ${ }^{5}$ ce même texte et qui comprend parfaitement le but que cherche à atteindre ce romancier sont à pied d'égalité au niveau du jeu d'imaginaire :

«La relation entre texte et lecteur décrite par $W$. Iser est faite d'attentes réciproques : le texte fait intervenir certains éléments d'un répertoire social et culturel plus ou moins connu du lecteur, et celui-ci projette sur le texte les intérêts et les dispositions qui lui sont propres ainsi que sa maîtrise du répertoire social et culturel.. ${ }^{6}$ Le texte littéraire chargé d'une intention esthétique tend à attirer l'attention du lecteur sur le répertoire social et culturel sur lequel se fonde le rapport auteur/lecteur. Si l'esthétique de la réception s'intéresse à la singularité de la rencontre avec l'œuvre littéraire et le lecteur en même temps, nous lisons chez Isabelle Kalinowski une vision d'ensemble globale pour cette approche littéraire lorsqu'elle ajoute que« l'esthétique de la réception s'élabore à partir de la perspective de l'œuvre et non de celle du lecteur. /) ${ }^{7}$

L'esthétique de la réception s'interroge sur la perspective de l'œuvre que l'auteur a voulu construire sur les différentes manières dont l'œuvre s'est

\footnotetext{
https://fac.umc.edu.dz/fll/images/cours-

fran\%C3\%A7ais/L3/L3\%20ETL\%20Yahia\%20Ouahmed.pdf, (consulté le 5 septembre 2021) 5- «La lecture subjective concerne en effet le processus interactionnel, la relation dynamique à travers lesquels le lecteur réagit, répond et réplique aux sollicitations d'une œuvre en puisant dans sa personnalité profonde, sa culture intime, son imaginaire.») Gérard Langlade, La lecture subjective, La littérature québécoise de 1970 à nos jours Numéro 145, printemps 2007, Disponible sur https://www.erudit.org/fr/revues/qf/2007-n145-qf1178006/47315ac.pdf.

6- Ibid., Rosmarin HEIDENREICH, La problématique du lecteur et de la réception, Cahiers de recherche sociologique, no 12, printemps 1989.

7- Cf. Isabelle Kalinowski. Hans-Robert Jauss et l'esthétique de la réception, Disponible sur https://doi.org/10.4000/rgi.649.
} 
emparée de ces perspectives esthétiques. En effet, si la perspective de l'œuvre est en lien étroit avec l'intention de l'auteur - qui permet de déterminer plus précisément l'interaction entre son œuvre et le lecteur il faut toutefois que la figure du lecteur soit explicitement inscrite dans l'œuvre.

On s'aperçoit tout particulièrement que la figure du lecteur reste plus ou moins au cœur du texte littéraire. Cela veut dire que l'acte de lecture est indissociable de l'acte d'écriture. Ces deux actes sont répartis pratiquement à parts égales entre l'auteur et le lecteur qui satisfont au mieux l'imagination littéraire. Celle-ci ne peut surgir que dans un décalage entre le besoin de l'auteur de produire un texte littéraire et la satisfaction des lecteurs qui sont susceptible de recevoir cette production littéraire.

Antoine Compagnon donne au rôle que joue la lecture une place considérable en affirmant que :

"L'expérience de la lecture, comme toute expérience humaine, est immanquablement une expérience double, ambiguë, déchirée : entre comprendre et aimer, entre la philologie et l'allégorie, entre la liberté et la contrainte, entre l'attention à l'autre et le souci de soi. Cette situation moyenne répugne aux vrais théoriciens de la littérature. ${ }^{8}$

En plus, nous constatons que la figure du lecteur demeure toujours sensible à la perception de l'évènement passé au moment de sa survenue qui peut être considéré comme une réception historique :

" Ainsi, si la figure du lecteur ne nous permet pas de contourner les problèmes posés par les théories de la réception (à savoir qu'il nous faut toujours effectuer des choix et restreindre notre point de vue, tout en sachant qu'il demeurera incomplet), elle permet 
néanmoins de faire évoluer le rôle du lecteur dans une autre direction, et nous fournit une perspective supplémentaire pour réfléchir à la littérature et à son appropriation. La figure, cette entité complexe, à la fois stable et malléable, analysable mais toujours en mouvement, se présente en effet comme un concept qui s'accorde bien avec la place du lecteur dans les théories littéraires : toujours présent, mais difficile à cerner.ı ${ }^{9}$

\section{Vers une réflexion sur la réception historique dans Samarcande}

La lecture approfondie de Samarcande montre une réflexion sur l'écriture de l'histoire et aussi sur les figures historiques. Notre roman est en face de l'histoire. Bien que l'auteur réveille l'évènement passé et l'histoire de cette évènement, il parvient aussi à éveiller notre intuition et notre sensibilité pour découvrir les sens, les émotions et pour avoir une vision d'ensemble sur cette réalité historique.

Hermann Broch a développé cette idée en affirmant que «l'histoire n'est pas seulement tenue d'élever le passé pour le faire entrer dans le 
présent, elle doit aussi abaisser le présent pour le faire entrer dans le passé afin qu'il devienne l'avenir du passé.ı) (Broch, 1966 : p.250'

Omar Khayyâm ${ }^{10}$ nous amène à réfléchir (1048 en Perse) sur la réception historique tourne autour de quelques histoires narrées dans Samarcande d'Amine Maalouf. La perception de ces figures se trouve développée en fonction de la civilisation et de la culture de l'Orient.

Notre romancier a mentionné les Robaiyat d'Omar Khayyâm (quatrains) au début du roman en disant :

«Peut-être en connaissez-vous le dénouement, les journaux l'ont rapporté à l'époque, certains ouvrages l'ont consigné depuis : lorsque le Titanic a sombré, dans la nuit du 14 au 15 avril 1912, au large de Terre-Neuve, la plus prestigieuse des victimes était un livre, exemplaire unique des Robaiyat d'Omar Khayyam, ${ }^{11}$ sage persan, poète, astronome.ı (Maalouf, 1988 : p.2)

Il est remarquable, en effet, que Samarcande, qui est reçu par le lecteur avec beaucoup de respect, annonce une sorte de réception historique qui se fonde, dans toute son extension, sur deux axes essentiels : les figures historiques et le discoure fictif.

Le discours fictif se coïncide tout au long du roman avec le temps et l'espace et aussi avec les mystères historiques qui résident dans cette ville légendaire. Par

10- Omar Khayyâm l'épopée de l'Islam, né le 18 mai 1048 à Nichapur en Perse (actuel Iran) où il est mort le 4 décembre 1131. Vu à travers la Perse et les yeux du philosophe, astronome et poète Omar Khayyâm, le long des XI e -XIIe siècles.

11- Omar Khayyam est connu pour ses rubä̈yat (quatrains). On appelle rubâi un genre particulier de quatrain persan dont les premier, deuxième et quatrième vers riment entre eux, tandis que le troisième est un vers blanc. En outre, les rubâïyat se font sur un rythme unique, spécial, toujours le même. Donc, au point de vue technique, le rubâi est soumis à des règles sévères qu'il faut observer à tout prix, sans quoi il n'y aurait plus de rubâi. Au point de vue poétique, les règles sont tout aussi rigides. Un rubâï est un petit poème complet qui doit exprimer une idée précise. En outre, il doit être clair, concis, très gracieux s'il traite un sujet galant, très profond s'il exprime une pensée philosophique. En un mot, le rubâi persan ressemble étrangement au sonnet français ; et les poètes persans qui ont produit de beaux rubâiiyat, sont aussi rares que les poètes français ayant réussi de parfaits sonnets. www 
ailleurs, Maalouf nous montre comment la narration fictive, mise en œuvre dans Samarcande, provoque une remise en question de la vérité historique :

"C'est d'un tel incident que va naître le manuscrit des Robaiyat, en l'été 1072. Omar Khayyam a vingt-quatre ans, il est depuis peu à Samarcande. $\{. .$.$\} Frais plaisir d'arpenter une ville inconnue, les yeux$ ouverts aux mille touches de la journée finissante : rue du Champ-deRhubarbe, un garçonnet détale, pieds nus sur les larges pavés, serrant contre son cou une pomme volée à quelque étalage; bazar des drapiers, à l'intérieur d'une échoppe surélevée, une partie de nard se dispute encore à la lumière d'une lampe à huile, deux dés jetés, un juron, un rire étouffé; arcade des cordiers, un muletier s'arrête près d'une fontaine, laisse couler l'eau fraîche dans le creux de ses paumes jointes, puis se penche, lèvres tendues, comme pour baiser le front d'un enfant endormi; désaltéré, il passe ses paumes mouillées sur son visage, marmonne un remerciement, ramasse une pastèque évidée, la remplit d'eau, la porte à sa bête afin qu'elle puisse boire à son tour.» (Maalouf, 1988 : p.2)

Cette vérité historique est un enjeu problématique qui parait évidemment non pas seulement dans la description détaillée d'Omar Khayyâm, mais aussi à travers le monde de ses passions pour cette ville légendaire. Cette description, qui se mêle à la narration, donne une vie authentique à cette vérité historique. Ce sens indique ce qui donne corps aux lieux où vivait ce grand poète.

La description de Samarcande souligne une interaction entre la ville d'un côté et la littérature, la culture de l'autre. Ceci nous a fait penser à la fréquence du mot ville dans la plupart des pages du roman. Cette fréquence a souvent le caractère d'une tendance thématique et d'une nécessité historique.

\section{Citons à titre d'exemple :}

«Frais plaisir d'arpenter une ville inconnue.» (Maalouf, 1988 : p.8) 
«Va dire à tous les ulémas de cette ville de venir dès l'aube se prosterner à mes pieds; la tête qui ne se courbera pas sera tranchée ; et que nul n'essaie de fuir, car aucune terre n'est à l'abri de ma colère »(Maalouf, $1988:$ p.27)

«Et soudain, dans l'obscurité de cette nuit, dans ce pavillon irréel, dans cette ville irréelle, te voici, femme belle, poétesse de surcroît, offerte. » (Maalouf, $1988:$ p.36)

"On peut penser qu'à l'époque les habitants d'une ville n'avaient aucune raison de préférer tel souverain turc à tel autre.n (Maalouf, $1988:$ p.52)

"Elle a juré que, si Malikshah franchissait le fleuve, elle lui interdirait à jamais sa couche et transformerait son harem en géhenne. Samarcande, ne l'oublions pas, est sa ville. ") (Maalouf, $1988:$ p.102)

«Chirine n’hésita pas à défier le mauvais sort et sa résidence fut désormais le cœur de la ville.») (Maalouf, 1988 : p.245)

Ce qui nous intéresse de plus en plus, concernant la réception historique, c'est l'alliance entre le fictif, l'histoire et l'authenticité. Le lecteur s'inspire des éléments caractéristiques de l'histoire qui se développent à cause de la double hybridité histoire/fiction et qui se trouvent accentuée par la représentation des figures historiques.

Tout cela incite le lecteur à découvrir les Robaiyat d'Omar Khayyam qui représentent la face authentique et lumineuse de l'Orient sous les yeux maaloufien. L'auteur a impressionné ses lecteurs par le vin et l'amour qui sont les grands inspirateurs de ses quatrains.

«Il faudrait attendre huit siècles avant que le monde ne découvre la sublime poésie d'Omar Khayyam, avant que ses Robaiyat ne soient vénérés comme l'une des œuvres les plus originales de tous 
les temps, avant que ne soit enfin connu l'étrange destin $d u$ manuscrit de Samarcande? » (Maalouf, $1988:$ p.18)

Nous allons découvrir le vin, les femmes, la vie, la sagesse et l'amour, à travers le manuscrit de ses quatrains. Ces thèmes sont inhérents au choix opéré par notre romancier. La perte de la trace du manuscrit de Samarcande pendant plusieurs siècles stimule Amine Maalouf à suggérer cette liberté d'esprit chez Omar Khayyâm malgré l'intolérance de la société dans laquelle il a vécu. Une traduction française des Robaiyat, qui a obtenu le prix de l'Académie Française en 1934, nous approche encore de l'esprit poétique, mystique et philosophique d'Omar Khayyâm:

«Un matin, une voix venait de la taverne. Elle criait :

A moi ! Joyeux buveurs, jeunes fous, levez-vous!

Venez encore vider une dernière coupe

Notre destin, de nous s'approche, le dernier vin est près de nous

Puisque personne ne pourrait te garantir le jour qui vient

Empresse-toi, soigne ton cœur, il s'abandonne à la tristesse

Bois avec moi, lune adorable. Lève et bois ta coupe vermeille

Là-haut, la lune dans le ciel plus jamais ne nous reverra.ı(Zadeh, 1934: p.83)

L'auteur nous fait plonger dans son univers fictif et historique, ce qui donne au lecteur l'occasion de gouter et de sentir ce que le romancier décrit. On y vit la réalité de la fiction à la fin du XIXe siècle à travers un personnage fictif qui s'appelle Benjamin le sage et qui joue deux rôles distincts dans le roman : un personnage principal et un narrateur qui raconte l'histoire du manuscrit original.

II est perdu au naufrage de Titani en 1912 et puis il s'embarque avec ce manuscrit. Nous sommes donc devant deux personnages qui 
appartiennent à deux temps différents : tandis que le premier personnage réel a retenu les points marquant de l'histoire du Xle siècle, le deuxième personnage, quant à lui, raconte I'histoire du XIXe siècle. Malgré cela, les deux personnages se croisent et leurs vies se confondent.

Notons que Robaiyat et l'histoire du manuscrit légendaire, qui occupent une place primordiale dans le domaine de la réception historique du roman, dominent la pensée du lecteur. Celui-ci se plonge dans I'histoire en cherchant le trésor du manuscrit qui se considère comme le fil conducteur de toute narration historique et de tous les évènements qui lient les autres histoires les unes aux autres

La question de la ville (Samarcande) en littérature indique le lieu de la narration et le thème d'ordre imaginaire, ce qui permet de percevoir au mieux le contour romanesque du texte en l'enrichissant du contexte référentiel dans lequel baigne le texte littéraire.

Jean-Claude Bationo, dans un article intitulé la ville objet de civilisation et de littérature en cours de français langue étrangère, a constaté que « la ville constitue un contenu civilisationnel, voire culturel, véhiculé par la

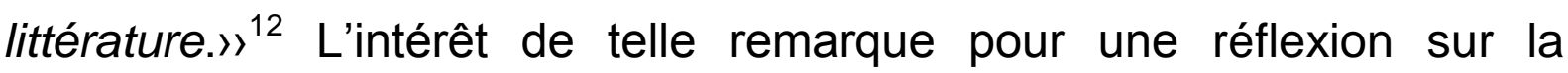
réception historique dans notre roman est de permettre d'appréhender la visée de la répétition du vocable Samarcande. C'est une figure de style qui est couramment utilisée dans toutes les pages du roman et parfois deux fois dans la même page. Citons à titre d'exemple:

" promène ton regard sur Samarcande! » (Maalouf, $1988:$ p.1) «C'est du kaghez chinois, le meilleur papier qui ait jamais été produit par les ateliers de Samarcande. " (Maalouf, 1988 : p.18)

12- Cf. Jean-Claude Bationo, R.Questions de communication, No12, La ville, objet de civilisation et de littérature en cours de français langue étrangère, Disponible sur https://doi.org/10.4000/questionsdecommunication.2405. 
« De Samarcande, on suit de près la bataille. » (Maalouf, 1988 : p. 50)

"Sais-tu quel est le vrai responsable des malheurs de Samarcande, et de tous nos malheurs ? » (Maalouf, $1988:$ p.101) «À Alamout, le Rédempteur ordonna que le Manuscrit de Samarcande soit vénéré comme un grand livre de sagesse. " (Maalouf, $1988:$ p.151)

" Il m’a expliqué qu'on appelait cet ouvrage le Manuscrit de Samarcande, qu'il contenait les quatrains écrits de la propre main du poète, auxquels avait été jointe en marge une chronique.») (Maalouf, 1988 : p.169)

" Du Manuscrit de Samarcande, plus un mot ; rien de personnel dans cette longue lettre, sinon, peut-être qu'elle commençait par " Cher ami lointain.ı) (Maalouf, 1988 : p.212)

" Je lui parlai du Manuscrit, des poèmes, de la chronique, des peintures qui évoquaient les amants de Samarcande. " (Maalouf, $1988:$ p.267)

" Après Samarcande, où aller ? Pour moi c'était l'extrême bout de l'Orient, le lieu de tous les émerveillements et d'une insondable nostalgie. » (Maalouf, 1988 : p.268)

II est remarquable que la ville de Samarcande permette au lecteur d'avoir recours à l'histoire, à la culture et à l'héritage de la civilisation musulmane à l'époque. Par ailleurs, Maalouf, dans Samarcande, a amalgamé d'une manière évidente entre la ville, la modernité, l'historicité et la littérature.

Comme l'écrit Jean Baudrillard «le discours de la ville, c'est la concurrence même : mobiles, désirs, rencontres, stimuli, verdict incessant des autres, érotisation continuelle, information, sollicitation 
publicitaire : tout cela compose une suite de destin abstrait de participation collective, sur un fond réel de concurrence généralisée.» (Baudrillard, $1970: 107$ )

Cela souligne que la ville de Samarcande se tient, sous toutes ses formes, aux échanges culturels et aux lieux privilégiés qui expliquent des faits historiques et sociaux, ce qui se considère comme des effets visibles.

Si nous nous interrogeons sur les figures historiques dans Samarcande telles qu'Omar Khayyâm, Hassan Sabbah et Nizam-El Molk, nous remarquons que l'évolution des personnages historiques dans le roman est indissociable du temps et de l'espace historique.

Samarcande comme roman historique ne se concentre pas seulement sur le passé, mais il mêle les personnages et les évènements historiques à l'imaginaire :

«Au fond de l'Atlantique, il y a un livre. C'est son histoire que je vais raconter.» (Maalouf, $1988:$ p.2)

Les liens entre texte littéraire et histoire dans l'œuvre d'Amine Maalouf constituent un défi soulevé par l'esthétique de la réception. À noter que notre romancier, qui se tourne vers la littérature avec ces deux premiers romans Les croisades vus par les arabes (1983) et Samarcande (1988) qui reflètent une réception litteraire et quelques figures historiques s'inspire des chroniqueurs arabes de l'époque et des historiens. Dans Samarcande d'Amine Maalouf, nous constatons une immense richesse de la pluralité historique et culturelle.

Si nous parlons de l'Orient du $\mathrm{XIX}^{\mathrm{e}}$ siècle et du début $d u \mathrm{XX}^{\mathrm{e}}$, nous parlons non pas seulement d'un voyage dans le temps et dans l'espace mais aussi d'un voyage et à travers l'univers historique et imaginaire. Cette histoire hybride de l'espace et du temps engendre une utilisation hybride du voyage dans le temps depuis aussi loin que Maalouf puisse 
imaginer. Et en raison de l'univers narratif et fictionnel hybride, notre romancier construit son propre parcours historique qui illustre clairement son ambition de créer un rapprochement entre l'orient et l'occident à travers plusieurs siècles :

" J'ai la profonde conviction que, si l'Orient ne parvient pas à se réveiller, bientôt l'occident ne pourra plus dormir.») (Maalouf, 1988 :2)

Arriver au terme de cette partie, nous pouvons constater que ces différents thèmes relatifs à l'univers historique et imaginaire montre la Perse d'Omar Khayyam, poète et libre penseur, mais aussi la Perse de Hassan Sabbah, fondateur de l'ordre des Assassins, la secte la plus redoutable de l'Histoire. C'est l'aventure d'un manuscrit né au XI e siècle et retrouvé six siècles plus tard.

\section{La réception de l'idée de l'univers imaginaire dans Samarcande}

Engager une réflexion sur la réception imaginaire, dans Samarcande d'Amine Maalouf, met en la valeur I'historicité romanesque qui conduit le cours des évènements vers une puissance imaginaire. Certains évènements et certains lieux ont un impact très puissant sur l'imaginaire du lecteur. Cette puissance imaginaire se porte sur le réel à travers un parcours fictif du manuscrit d'Omar Khayyâm.

Le thème de l'imaginaire qui se coïncide avec le réel se présente au niveau de la forme textuelle comme une écriture romanesque qui fait surgir fréquemment des images et des souvenirs. C'est ainsi que nous pouvons affirmer que le développement de l'imagination selon Amin Maalouf est lié à un cheminement de la pensée sur son univers imaginaire. 
Ce cheminement cherche à s'interroger sur le sens de l'univers imaginaire et à questionner les grandes dimensions de la pensée narrative. Notons que le réel et l'imaginaire se rencontrent pour accorder aux évènements du roman une réception à la fois réaliste et fictive. Le roman commence en juin 1072 et se terminer le 15 mars 1912 dans le même temps que les images se développent dans Samarcande dès le début jusqu'à la fin du roman.

La question qui se pose est la suivante: A quoi servent les imaginaires historiques dans Samarcande ? Samarcande aura peut-être à penser et à expérimenter le monde à l'épreuve de notre imagination. En se focalisant sur la construction imaginaire, nous constatons que Maalouf fait appel à des concepts offrant une grande variété stylistique pour marquer son imagination à travers ses écrits romanesques et surtout à travers ses romans historiques comme Léon l'Africain (1986) et Samarcande (1988).

Si l'imagination se considère comme une instance centrale dans l'ouvre de Maalouf, l'univers imaginaire sera réévalué comme une source d'expériences individuelles et collectives. Cela veut dire que l'imaginaire, selon G.Durant se porte sur les images, les symboles et les mythes qui nourrissent le champ de notre expérience individuelle et collective.

Amine Maalouf, de sa part, s'occupe au développement de cet imaginaire qui nous permet d'évoquer le passé pour rendre présent ce qui est éloigné et pour intégrer ce passé dans une histoire très loin qui nous présente une perception contemporaine.

Le grand intérêt de la réception imaginaire réside dans le rôle du narrateur fictif, nommé Benjamin Omar Lesage, un narrateur fictif qui prend en charge l'histoire du manuscrit de Samarcande, notant qu'aucun original des Robaïyat n'a jamais été retrouvé. L'histoire de ce manuscrit 
présente ainsi deux aspects semblables, celui du réel et celui de l'imaginaire.

" Qu'il faudrait attendre huit siècles avant que le monde ne découvre la sublime poésie d'Omar Khayyam, avant que ses Robaiyat ne soient vénérés comme l'une des œuvres les plus originales de tous les temps, avant que ne soit enfin connu l'étrange destin du manuscrit de Samarcande ?» (Maalouf, $1988: 18$ )

Dans Samarcande, le narrateur fait référence à la personnalité d'Omar Khayyâm à partir de la première page du roman, ensuite lorsque le narrateur déplore la perte du manuscrit de Khayyâm, il le présente comme «sage persan, poète, astronome.

Benjamin Omar Lesage, le principal narrateur, et sa femme, la princesse Chirine personnage fictif aussi, embarquent sur le Titanic en direction des Etats-Unis pour garder le Manuscrit d'Omar Khayyâm. Le narrateur de Samarcande, Benjamin Omar Lesage raconte I'histoire d'un livre noyé au fond de l'Atlantique.

II se présente pour la première fois dans le roman: «Mon nom est Benjamin Lesage, je viens de la part d'Henri Rochefort.») (Maalouf, 1988 :175)

Henri Rochefort est le fil conducteur de lancer cette narration fictive afin d'évoquer les trois personnages, Nizam-el-Molk, Omar Khayyam et Hassan Sabbah. Les trois amis sont les trois Persans qui ont marqué, chacun à sa façon, les débuts de notre millénaire.

Le narrateur présente les trois personnages en citant: «je vous suis reconnaissant de m'arracher quelques instants à mes pénibles préoccupations. Le sujet que vous évoquez m'a toujours passionné. Avez-vous lu dans l'introduction de M. Nicolas aux Robaiyat l'histoire des 
trois amis, Nizam-el-Molk, Hassan Sabbah et Omar Khayyam ? ") (Maalouf, $1988: 177$ )

Le premier, Nizam-el-Molk, est le plus riche, le plus puissant et le plus âgé. Le deuxième, Omar khayyam, un homme de savoir qui a une vision philosophique sur le monde. Le troisième, Hassan Sabbah, un homme de croyance. Les expériences de ces personnages historiques sont présentées par une narration fictive afin de faire intervenir le lecteur dans un monde qui vient sur le même pied d'égalité avec le monde romanesque de l'auteur.

Le lecteur se trouve entre l'imaginaire et le réel, entre le narrateur fictif Benjamin Omar Lesage et le vrai poète Omar Khayyam. Ce carrefour leur permet de se rencontrer et de tracer un chemin imaginaire de l'histoire du manuscrit.

\section{Conclusion}

Dans cet article, nous avons proposé de mettre en évidence les principales caractéristiques du langage romanesque d'Amin Maalouf à caractère historique et imaginaire qui alimentent son roman. Le roman de Samarcande que nous avons choisi pour cet article nous a permis de mettre en évidence les trois thèmes essentiels : la réception littéraire, la réception historique et la réception imaginaire. Ce choix témoigne de l'importance du rôle joué par Maalouf qui se considère comme un alchimiste qui a réussi à transformer ses impressions narratives en image et à reconstruire l'identité arabe à partir de l'identité culturelle en stimulant l'imagination du lecteur. 
Ceci dit, nous pouvons noter qu'Amin Maalouf est parmi les rares écrivains qui sont parvenu à tracer son chemin de réussite en s'inscrivant dans le vaste champ de la littérature contemporaine. Ses romans constituent une base vitale du débat sur l'hybridité de genres qui ne s'exprime pas seulement au niveau de la forme ou du fond, mais aussi au niveau de l'organisation du texte romanesque.

Nous terminons cet article sur l'idée que Maalouf provoque chez le lecteur une sorte de va et vient entre l'imaginaire et le réel. Notre romancier a réussi à garder le fil de l'histoire raconté par ses personnages historiques et à mettre le lecteur face à une fin émouvante qui lui donne envie de lire et relire ses œuvres. Amin Maalouf cherche à instaurer un rapport entre le texte le lecteur. C'est la cause pour laquelle son style est devenu l'une des plumes prestigieuses de la littérature contemporaine.

\section{Références bibliographiques :}

\section{Corpus}

Amin Maalouf, Samarcande. J.-C. Lattès, Paris, (1988).

Webographie :

https://doi.org/10.4000/questionsdecommunication.2405

https://www.erudit.org/fr/revues/crs/1989-n12-crs1516226/1002059ar.pdf https://www.grainesdepaix.org/fr/ressources/dictionnaire/identite culturell $\underline{e}$

https://www.universalis.fr/encyclopedie/reception-art-et-litterature/ 
https://oic.uqam.ca/sites/oic.uqam.ca/files/documents/04.valerie-coolslecteur-en-theorie.pdf

https://fac.umc.edu.dz/fll/images/cours-

fran\%C3\%A7ais/L3/L3\%20ETL\%20Yahia\%200uahmed.pdf

https://www.erudit.org/fr/revues/qf/2007-n145-gf1178006/47315ac.pdf

https://doi.org/10.4000/rgi.649

\section{Ouvrages et Articles de revues :}

E'Tessam-Zadeh, (1934), Omar Khayyem., Les Rubayat, traduction du persan, Editeur. Maurice d'Hartoy Paris.

Émery-Bruneau, (2010), Le rapport à la lecture littéraire. Des pratiques et des conceptions de sujets-lecteurs en formation à l'enseignement du français à des intentions didactiques, Thèse de doctorat, Université Laval, Québec.

Hans Robert Jauss, (1978), Pour une esthétique de la réception, Paris, NRF, Gallimard.

Isabelle Kalinowski. Hans-Robert Jauss et l'esthétique de la réception, p.151

Jean Baudrillard, (1970), La Société de consommation, Paris, Gallimard. COMPAGNON A. (1998) Le Démon de la théorie, littérature et sens commun, Paris, Seuil, p. 194.

Josias Semujanga, Protée, (1999), La réception, volume 27, numéro 2.

Nicolas Xanthos, (1999), Protée, LA LECTURE LITTÉRAIRE COMME PARCOURS DANS L'AIRE DU DIRE. Volume 27, numéro 2. 
التلقي التاريخي والتخيلي في رواية سمرقند عند أمين معلوف

\author{
د. كامل محمد منير \\ كلية الآداب_جامعة المنوفية
}

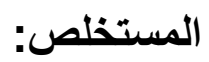

يتناول هذا البحث العالَم التاريخي والتخيلي في رواية سمرقند (9/1 () لأمين معلوف. ولكي نوضح موقف معلوف بالنسبة الى تاريخ الشرق، يمكننا القول إنه عندما يتحدث عن تاريخ بلد ما أو تاريخ رجل ما أو تاريخ شاعر ما، فإنه يسلِّط الضوء على عالمه التخيلي. يهدف هذا المقال إلى تحليل مفهوم التلقي التاريخي والتخيلي دن خلال الوقائع التاريخية المحكية، وبالتالي سيتيح لنا هذا التحليل فهم التداخل الثقافي بين الثرق و الغرب. اننا نعتقد أن هناك بعض المفاهيم الأساسية التي تلعب دورًا هاما في رواية سمرقند منها التسامح و النبيذ والثعر والعنف. ان هذا البحث يُعتبر جزء لا يتجزأ من منظورنا الذي يقترح وضع حلول للقضايا الثقافية والأيديولوجية والفلسفية. ان ما يهمنا في هذا البحث هو محاولتنا فهم الكيفية التي يستخدمها معلوف لعمل مزيجًا متوازنًا بين المدلول التاريخي والمعنى الوهمي، حيث يمكن ملاحظة أن معلوف نجح في بناء عالمه السردي الواقعي والخيالي. أخيرًا، سنتناول في هذا البحث المكون الروائي في رواية سمرقند عند أمين معلوف الذي يهتم بشكل أساسي بالمصادر التاريخية للثرق.

الكلمات الإفتتاحية، التلقي التاريخي، التلقي التخيلي، تاريخ الثرق والغرب، التفاعل التقافي 